\title{
EXTENDING MONOTONE DECOMPOSITIONS OF MANIFOLDS
}

\author{
JOHN J. WALSH
}

\begin{abstract}
Let $M^{m}(m \geqslant 3)$ be a compact, connected PL manifold and let $X \subseteq M$ be a proper, closed subset of the interior of $M$ such that for each open, connected subset $U \subseteq M$ either $U-(X \cap U)$ is connected or $X \cap$ $\operatorname{bd}(U) \neq \varnothing$. Let $P$ be a connected and simply connected polyhedron with $\operatorname{dim} P>3$. There exists a monotone mapping $f$ from $M$ onto $P$ with each component of $X$ being a point-inverse of $f$. In the case with $M$ oriented and $P$ the $\boldsymbol{m}$-sphere, there exists such a monotone mapping of each degree.
\end{abstract}

1. Introduction. Bing [Bi; $m=3$ ], Bean [Be; $m=3$ ], and Coram [Co; $m \geqslant 4$ ] showed that if $X$ is a proper, closed subset of a PL manifold $M^{m}$ such that for each open, connected subset $U \subseteq M$ either $U-(X \cap U)$ is connected or $X \cap \operatorname{bd}(U) \neq \varnothing$, then there is a monotone mapping $f$ from $M$ onto the $m$-sphere with each component of $X$ being a point-inverse of $f$. These results are generalized in two ways. First, if $P$ is a simply connected polyhedron with $\operatorname{dim} P \geqslant 3$, then there is a monotone mapping $f$ from $M$ onto $P$ with each component of $X$ being a point-inverse of $f$. Second, if $M$ is oriented, then there is such a monotone mapping onto the $m$-sphere of each degree. The mappings constructed by Bing, Bean and Coram have degree \pm 1 ; PL monotone mappings between manifolds of the same dimension have degree \pm 1 and their mappings are "almost" PL.

In general, the assumption that $P$ be simply connected cannot be omitted since monotone mappings between ANR's induce surjections between fundamental groups. Adopting a point of view used in [Wa-1], [Wa-2], a generalization to the nonsimply connected case is possible; such a generalization will appear in a subsequent paper.

Since there are limitations placed on those compacta which are monotone images of 2-dimensional manifolds [Ho], [Mo], [R-S], [Yo], the assumption that $M^{m}$ have dimension at least three is essential. However, the first result holds for certain simply connected 2-dimensional polyhedra and, if $X$ is connected, for simply connected 1-dimensional polyhedra.

The main tool used in the proofs of the above results is an extension theorem in [Wa-3]; roughly, the theorem says that if a mapping from the boundary of an $m$-manifold $(m \geqslant 3)$ to a simply connected ANR has an

Received by the editors February 8, 1978 and, in revised form, May 30, 1978.

AMS.(MOS) subject classifications (1970). Primary 57A60; Secondary 54B15, 54C10, 57A15. 
extension to the entire manifold, then it has an extension which is monotone.

2. Definitions and basic concepts. Spaces are assumed to be separable and metric; we use $\partial M$ to denote the boundary of a manifold and bd( ), int( ), cl( ), and $\operatorname{diam}($ ) to denote the topological boundary, topological interior, closure, and diameter, respectively. A mapping is a continuous function; a mapping is monotone $\left(=U V^{\circ}\right)$ if each point-inverse is compact and connected. Let $Q$ be a collection of sets; $Q^{*}$ denotes the union of the elements of $Q$.

Let $M^{m}(m \geqslant 3)$ be a compact, connected PL manifold and let $X \subseteq(M$ $-\partial M)$ be a proper, closed subset of $M$ such that for each open connected subset $U \subseteq M$ either $U-(U \cap X)$ is connected or $\operatorname{bd}(U) \cap X \neq \varnothing$. Then there is a sequence $\left\{\mathscr{N}_{i}\right\}_{i=1}^{\infty}$ satisfying: (1) $\mathfrak{K}_{i}=\left\{M_{i}^{1}, \ldots, M_{i}^{q_{i}}\right\}$ is a finite collection of pairwise disjoint compact $m$-dimensional PL submanifolds of $M-\partial M$ with connected boundaries; (2) each $M_{i}^{j}$ is contained in the interior of some $M_{i-1}^{k}$; (3) $\cap_{i=1}^{\infty} \mathfrak{N}_{i}^{*}=X$. The sequence $\left\{\mathscr{N}_{i}\right\}_{i=1}^{\infty}$ is called a special defining sequence for $X$.

The next result is an immediate corollary to [Wa-3; Main Theorem] and is the "key" result needed in this paper.

2.1. TheOREM. Let $M^{m}(m \geqslant 3)$ be a compact, connected PL manifold and let $P$ be a compact, simply connected polyhedron. Each mapping from $M$ to $P$ is homotopic rel $\partial M$ to a monotone mapping from $M$ onto $P$.

3. Basic extension theorem. This section contains the proof of the following result on extending monotone decompositions; the proofs of subsequent results rely heavily on the proof.

3.1. TheOREM. Let $M^{m}(m \geqslant 3)$ be a compact, connected PL manifold, let $P$ be a compact, simply connected polyhedron with $\operatorname{dim} P \geqslant 3$, and let $X \subseteq(M$ $-\partial M)$ be a proper, closed subset such that for each open connected subset $U \subseteq M$ either $U-(U \cap X)$ is connected or $\operatorname{bd}(U) \cap X \neq \varnothing$. There is a monotone mapping $f$ from $M$ onto $P$ with each component of $X$ being a point-inverse of $f$.

Proof. Let $\mathfrak{T}_{i}=\left\{M_{i}{ }^{1}, \ldots, M_{i}^{q_{i}}\right\}, i=1,2, \ldots$, be a special defining sequence for $X$ with $\mathfrak{T}_{1}=\left\{M_{1}^{1}\right\}$. Let $n=\operatorname{dim} P$ and let $B \subseteq P$ be a PL $n$-ball. For $i=1,2, \ldots$, let $\mathscr{B}_{i}=\left\{B_{i}^{1}, \ldots, B_{i}^{q_{i}}\right\}$ be a collection of pairwise disjoint PL $n$-balls with diameters less than $1 / i$ such that $B_{1}^{1} \subseteq(B-\partial B)$ and $B_{i}^{j} \subseteq\left(B_{i-1}^{k}-\partial B_{i-1}^{k}\right)$ if and only if $M \subseteq\left(M_{i-1}^{k}-\partial M_{i-1}^{k}\right)$.

Since $P$ is simply connected and $n \geqslant 3$, each of the sets $\operatorname{cl}\left(P-B_{1}^{l}\right)$ and $\operatorname{cl}\left(B_{i}^{j}-\mathscr{B}_{i+1}^{*}\right)$ is simply connected, and since the $\mathfrak{T}_{i}$ 's form a special defining sequence, each of the sets $\operatorname{cl}\left(M-M_{1}^{1}\right)$ and $\operatorname{cl}\left(M_{i}^{j}-\mathfrak{N}_{i+1}^{*}\right)$ is connected. Let $f^{\prime}$ be a mapping from $\cup\left\{\partial M_{i}^{j}: i \geqslant 1\right.$ and $\left.1 \leqslant j \leqslant q_{i}\right\}$ to $\cup\left\{\partial B_{i}^{j}: i \geqslant 1\right.$ and $\left.1 \leqslant j \leqslant q_{i}\right\}$ such that $f^{\prime}$ maps $\partial M_{i}^{j}$ onto $\partial B_{i}^{j}$ and is null-homotopic (as a mapping from $\partial M_{i}^{j}$ to $\partial B_{i}^{j}$ ). The mapping $f^{\prime}$ from $\partial M_{i}^{j}$ to 
$\partial B_{i}^{j}$ can be defined by letting $f^{\prime}=\alpha \circ \beta$ where $\beta$ maps $\partial M_{i}^{j}$ onto the unit interval $I$ and $\alpha$ maps $I$ onto $\partial B_{i}^{j}$.

Since $f^{\prime}$ is null-homotopic, $f^{\prime}$ extends to a mapping from $\operatorname{cl}\left(M-M_{1}^{1}\right)$ to $\operatorname{cl}\left(P-B_{1}^{1}\right)$ and, therefore, Theorem 2.1 implies that $f^{\prime}$ extends to a monotone mapping from $\operatorname{cl}\left(M-M_{1}^{1}\right)$ onto $\operatorname{cl}\left(P-B_{1}^{1}\right)$. Similarly, $f^{\prime}$ extends to a monotone mapping from $\operatorname{cl}\left(M_{i}^{j}-\mathfrak{N}_{i+1}^{*}\right)$ onto $\operatorname{cl}\left(B_{i}^{j}-\mathscr{S}_{i+1}^{*}\right)$. Finally, extend $f^{\prime}$ to a mapping $f$ from $M$ onto $P$ by letting $f\left(\cap_{i=1}^{\infty} M_{i}^{j_{i}}\right)=\bigcap_{i=1}^{\infty} B_{i}^{j_{i}}$ for each nested collection $M_{1}^{j_{1}} \supseteq M_{2}^{j_{2}} \supseteq \ldots$. The mapping $f$ is the desired monotone mapping from $M$ onto $P$.

4. Extensions of different degrees. In this section, assuming that $M^{m}$ is orientable and without boundary and that $P$ is the $m$-sphere, it is shown that there is a monotone mapping $f$ as in Theorem 3.1 of each degree. The monotone mapping constructed in $\S 3$ is easily seen to have degree zero.

4.1. TheOREM. Let $M^{m}(m \geqslant 3)$ be a compact, connected oriented $P L$ manifold without boundary and let $X \subseteq M$ be a proper closed subset such that for each open connected subset $U \subseteq M$ either $U-(U \cap X)$ is connected or $X \cap \operatorname{bd}(U) \neq \varnothing$. There exists a monotone mapping $f$ of each degree from $M$ onto the $m$-sphere $S^{m}$ with each component of $X$ being a point-inverse of $f$.

Proof. Following the notation from the proof of Theorem 3.1, define $f^{\prime}$ so that $f^{\prime}: \partial M_{i}^{j} \rightarrow \partial B_{i}^{j}$ is onto and has degree $d$. Since $\operatorname{cl}\left(S^{m}-B_{1}^{1}\right)$ is an $m$-ball, $f^{\prime}$ extends to a mapping from $\operatorname{cl}\left(M-M_{1}^{1}\right)$ to $\operatorname{cl}\left(S^{m}-B_{1}^{1}\right)$; Theorem 2.1 guarantees that the extension can be chosen to be monotone and onto. If $f^{\prime}$ extends to a mapping from $\operatorname{cl}\left(M_{i}^{j}-\Re_{i+1}^{*}\right)$ to $\operatorname{cl}\left(B_{i}^{j}-\Re_{i+1}^{*}\right)$, then Theorem 2.1 implies that each such extension can be chosen to be monotone and onto. An argument using the Mayer-Vietoris sequence would show that the induced mapping has degree $d$. Lemma 4.3 below guarantees that the necessary extensions of $f^{\prime}$ exist.

In the following lemmas, it is necessary to make appropriate choices of orientations for the various boundary components.

4.2. Lemma. Let $B=S^{m}-\cup_{i=1}^{n} \operatorname{int}\left(B_{i}\right)$ where the $B_{i}$ 's are pairwise disjoint $P L m$-balls and let $d$ be an integer. If $m \geqslant 3$, then there is a mapping $f$ : $\left(B ; \partial B_{1}, \ldots, \partial B_{n}\right) \rightarrow\left(B ; \partial B_{1}, \ldots, \partial B_{n}\right)$ with $f: \partial B_{i} \rightarrow \partial B_{i}$ having degree $d$ for each $i=1, \ldots, n$.

Proof. We assume that $m=3$, since the higher dimensional cases are proved in a similar manner. Let $f^{\prime}: S^{3} \rightarrow S^{3}$ be a mapping which is the second suspension of a degree $d$ mapping from $S^{1}$ to itself. There are pairwise disjoint PL 3-balls $B_{1}^{\prime}, \ldots, B_{n}^{\prime}$ (centered on the suspension circle) with $f^{\prime}$ : $\left(B_{i}^{\prime}, \partial B_{i}^{\prime}\right) \rightarrow\left(B_{i}^{\prime}, \partial B_{i}^{\prime}\right)$ and with $f^{\prime}: \partial B_{i}^{\prime} \rightarrow \partial B_{i}^{\prime}$ having degree $d$. The mapping $f$ is obtained by conjugating $f^{\prime}$ with an appropriate homeomorphism of $S^{3}$ and restricting the mapping to $B$. 
4.3. LEMMA. Let $B=S^{m}-\cup_{i=1}^{n} \operatorname{int}\left(B_{i}\right)$ where the $B_{i}$ 's are pairwise disjoint $P L$ m-balls, let $M^{m}$ be a compact, connected, oriented PL manifold with the boundary of $M$ having components $\partial M_{1}, \ldots, \partial M_{n}$, and let $d$ be an integer. If $m \geqslant 3$, there is a mapping $f:\left(M ; \partial M_{1}, \ldots, M_{m}\right) \rightarrow\left(B ; \partial B_{1}, \ldots, \partial B_{n}\right)$ with $f:$ $\partial M_{i} \rightarrow \partial B_{i}$ having degree $d$.

Proof. It suffices to consider the case with $d=1$, since the other cases follow by composing with the mappings obtained in Lemma 4.2. Let $T_{1}, \ldots, T_{n-1}$ (respectively, $T_{1}^{\prime}, \ldots, T_{n-1}^{\prime}$ ) be pairwise disjoint tubes-i.e., relative regular neighborhoods of arcs-such that $T_{i}$ (respectively, $T_{i}^{\prime}$ ) connects $\partial B_{i}$ and $\partial B_{n}$ (respectively, $\partial M_{i}$ and $\partial M_{n}$ ). Let $f^{\prime}$ be a mapping from $\left(\cup_{i=1}^{n} \partial M_{i}\right) \cup\left(\cup_{i=1}^{n-1} T_{i}\right)$ to $\left(\cup_{i=1}^{n} \partial B_{i}\right) \cup\left(\cup_{i=1}^{n-1} T_{i}\right)$ with $f^{\prime}: \partial M_{i} \rightarrow \partial B_{i}$ having degree 1 and with $f^{\prime}: T_{i}^{\prime} \rightarrow T_{i}$ a homeomorphism. Since $\operatorname{cl}(B-$ $\cup_{i=1}^{n-1} T_{i}$ ) is an $n$-ball, $f^{\prime}$ extends to the desired mapping $f$.

5. One and two dimensional cases. The following are versions of Theorem 3.1 which hold when $P$ is a 1- or 2-dimensional polyhedron.

5.1. Theorem. Let $M^{m}(m \geqslant 3)$ and $X$ be as in Theorem 3.1 and let $P$ be a compact, simply connected 2-dimensional polyhedron such that when $P$ is triangulated some 2-simplex has a free face. There is a monotone mapping $f$ from $M$ onto $P$ with each component of $X$ being a point-inverse of $f$.

Proof. Let $B \subseteq P$ be a 2-simplex (in some triangulation of $P$ ) with $\partial B$ containing an arc $A$ which is a free face. Following the notation in the proof of Theorem 3.1, choose the $\mathscr{B}_{i}$ 's such that $B_{1}^{1} \subseteq B-\operatorname{bd}(B), B_{i}^{j} \subseteq\left(B_{i-1}^{k}-\right.$ $\left.\operatorname{bd}\left(B_{i-1}^{k}\right)\right)$ if and only if $M_{i}^{j} \subseteq\left(M_{i-1}^{k}-\partial M_{i-1}^{k}\right)$, and $\partial B_{i}^{j} \cap A$ is an arc. The important feature is that each of the sets $\operatorname{cl}(P-B)$ and $\operatorname{cl}\left(B_{i}^{j}-\mathscr{B}_{i+1}^{*}\right)$ is simply connected.

5.2. TheOREM. Let $M^{m}(m \geqslant 3)$ and $X$ be as in Theorem 3.1 with the additional assumption that $X$ be connected and let $P$ be a compact, simply connected 1-dimensional polyhedron. There is a monotone mapping $f$ from $M$ onto $P$ with $X$ being a point-inverse of $f$.

Proof. The polyhedron $P$ is necessarily a finite tree, the proof of Theorem 3.1 applies provided the $\mathscr{B}_{i}$ 's are chosen so that $\cap \mathscr{B}_{i}{ }^{*}$ is an endpoint of $P$.

\section{BIBLIOGRAPHY}

[Be] R. J. Bean, Repairing embeddings and decompositions in $S^{3}$, Duke Math. J. 36 (1969), 379-385. MR 39 \#4820.

[Bi] R. H. Bing, Extending monotone decompositions of 3-manifolds, Trans. Amer. Math. Soc. 149 (1970), 351-369. MR 41 \# 7656.

[Co] D. Coram, Semicellularity, decompositions, and mappings in manifolds, Trans. Amer. Math. Soc. 191 (1974), 227-244.

[Ho] J. G. Hocking, Approximations to monotone mappings on noncompact two dimensional manifolds, Duke. Math. J. 21 (1954), 639-651. MR 16, 158.

[Mo] R. L. Moore, Concerning upper semi-continuous collections of continua, Trans. Amer. Math. Soc. 27 (1925), 416-428. 
[R-S] J. H. Roberts and N. E. Steenrod, Monotone transformations of 2-dimensional manifolds, Ann. of Math. (2) 39 (1938), 851-862.

[Wa-1] J. J. Walsh, Monotone and open mappings on manifolds. I, Trans. Amer. Math. Soc. 209 (1975), 419-432.

[Wa-2] _ Monotone and open mappings onto ANR's, Proc. Amer. Math. Soc. 60 (1976), 286-288.

[Wa-3] _ Extending mappings to monotone mappings, Houston J. Math. 3 (1977), 579-592.

[Yo] J. W. T. Youngs, Homeomorphic approximations to monotone mappings, Duke Math. J. 41 (1948), 87-94.

Department of Mathematics, University of TennesSee, KNOXVIlle, TenNesSee 37916 